\title{
BMJ roundtable debate: How can we get better at providing patient centred care?
}

\author{
Participants in our discussion on person centred care in January agreed that a change in culture \\ and better use of technology could benefit both patients and doctors, Emma Parish reports
}

\section{Emma Parish editorial registrar, The BMJ}

\begin{abstract}
When the news is filled with stories of overflowing emergency departments, understaffed hospitals, and long delays in timely treatment for patients, where is the focus on providing care which centres on people's needs and priorities?

We have been talking about the importance of person centred care for decades, and frustration at the slow pace of change on the ground was tangible among the patients, clinicians, and patient advocates invited by The BMJ to discuss the topic.

Angela Coulter, from the Informed Medical Decisions Foundation, acknowledged that, "most nurses, most clinicians do their utmost to treat people with dignity and respect and to listen to them," but underlined that in person centred care "we're talking about something that's more ambitious than that." Alf Collins, clinical associate in person centred care at the Health Foundation, pointed out that all medical care must show people "dignity, compassion and respect." But for it to be person centred services must also be well coordinated and structured in a way that supports and empowers people to take charge of their health and long term conditions.
\end{abstract}

Providing people with the support they need to cope with illness and manage their health is not a luxury but a necessity, participants agreed, and it cannot be fully achieved without restructuring traditional models of healthcare.

"Physician centred models" that have subverted patient centredness must be abandoned, said Rupert Whitaker of the Tuke Institute. Major reorganisation of services needs patient engagement and involvement, and this means a cultural change.

Sara Riggare, who has Parkinson's disease, made the point that she (along with millions of others who live with long term conditions) is already in charge of her health. "I spend one hour per year with my neurologist and 8765 hours in self care." The system must recognise what patients do for themselves, she said, and all agreed that inflexible paternalistic systems must change if they are to successfully support self care.

There was consensus that culture change is well overdue and that strong leadership of clinical teams and reform of medical education is essential. Coulter acknowledged the "major advances in undergraduate training" but emphasised that the "problem comes in postgraduate" education. She called on the royal colleges to take responsibility for leading change to ensure the principles of person centred care were implemented. Reaching a point where clinicians can feel able to admit "they can't and don't know everything" is, it was suggested, fundamental to changing the perception of how people participate in their own care.

The inflexibility of the current structure of healthcare is a problem that both patients and doctors struggle with, said Michael Seres, who has Crohn's disease. Many people are left navigating the system alone. "If you want to manage your complex conditions, you do it your way and your healthcare providers have to work round you." Ben Mearns, a consultant in acute care and elderly medicine, agreed: "If we' re truly going to be person centred I think we've got to move away from primary and secondary and tertiary [care] ... you have to put the person in the centre of the process and then allow all of our systems to [provide the] resource the patient or the person [needs]." The current "factory-style way of processing patients" has been to the detriment of good person centred care, he argued.

\section{Change the medical culture and create new models of care with patients}

Ideally, each contact with healthcare would be with the providers best suited to meet an individual's needs. One example cited was people who have behavioural or mental health disorders and social problems. Whitaker, proposed, "you need to have a team which includes nurse, psychologists, clinical social worker so that those issues are addressed fully and competently because they're key to helping a person get well and stay well and not need to come back."

Amir Hannan, a general practitioner, said we should question why emergency departments seem to be the first choice for people seeking care. Jeremy Taylor, from National Voices, suggested that it was not just a question of being unable to access care elsewhere. It reflected people's genuine concern about their 
health, and knowing more about their worries and their expectations would help shape the delivery of services. Several participants at the roundtable made the point that an underappreciated part of a clinician's role is to reassure. Rosamund Snow, The BMJ's patient editor, said, "If you're seeing a worried but well person, you're doing your job."

Coproduction of healthcare was widely seen as a positive step, with patients and clinicians "sharing the work." Some argued that "patients are on the team but just not recognised." Seres spoke of the "real role and great responsibility" of patients. As a patient, he said, it is "easy to pass the buck onto the clinicians and the professionals," but if you do "you don't have a partnership, and you don't have a relationship unless both parties work at it."

Alex Silverstein, past president of the International Diabetes Federation's young leaders in diabetes project, referred to the "enormous disconnect" between patients and clinicians and agreed with the need to determine "active shared goals." Determining these goals, recording them, and measuring whether they have been reached is important. Hannan discussed an initiative used in his practice where patients pledge their own health related goal as part of NHS Change Day. This is recorded in the medical notes and revisited in future consultations. Doing this provides a sense of "ownership" and promotes a "partnership of trust," he said. He also urged greater patient involvement in commissioning services.

Taylor spoke of the value of harnessing the collective patient voice to push for new and better designed services. Rigarre agreed. All patients, she underlined, stand to benefit from the advocacy of patient groups and the efforts of individual patient leaders.

An important warning note was sounded by some that any advances in patient empowerment must ensure the less vocal and more vulnerable are not excluded. Too many people, Coulter warned, still feel that they are on their own and they "don't belong to patient support groups." Participants agreed that clinicians should see it as a priority for "everyone to be empowered." The default position should be to assume all patients have capacity to make decisions about their care.

\section{New technologies, better relationships}

The use of new technologies to engage and empower people was discussed. A show of hands around the table found many people were using wearable activity trackers and mobile technology to monitor their health. Paul Wicks, from
PatientsLikeMe, drew attention to the fact that many people now use the internet as "pre-primary care."

The academic community need to realise "that's where the action is," agreed Paul Hodgkin, a former general practitioner and founder of Patient Opinion. "Patients have a voice now, and it's a public voice. They've got Twitter, they've got Facebook ... PatientsLikeMe, support groups" and so "they've got solidarity." If more clinicians were to see the benefit of these already available resources for patients, including peer to peer support, and direct people to them, it might give more time to focus on those who need immediate attention.

Amid talk of system change and mindset shifts, participants were encouraged to come up with practical things that could be done now to advance person centred care. The list was long and included letting patients email clinicians, get results of routine blood tests texted to them, and using teleconferencing more widely (box). However, participants agreed that we should guard against moving to a "consumer" focused system.

Knowing about ways that promote person centred care is important, and so is incentivising them. "We've got measures to determine if person centred care is delivered. We just need to use them." emphasised Coulter. There was broad agreement that following the principle "what gets measured gets done," doing this for person centred care would enable it to be prioritised by healthcare providers.

But ultimately, participants agreed, "healthcare is about relationships." If we want to improve the quality of healthcare, and people's experience of it, we need to manage expectations and bridge the divide between physicians and patients. As Silverstein said, "we'll only get to a partnership model when we reach a common position that what patients want and what GPs [and other doctors] want are actually the same thing."

An edited podcast from the round table and additional comment from international discussants (Dominck Frosch associate professor, University of California; Rakhal Gaitonde chair, community advisory board of the National Institute for Research on Tuberculosis; Cristin Lind, patient advocate, Rare Diseases Sweden; Maggie Breslin US designer, researcher and writer; Matthew Maleska, designer, Patient Revolution Project; Jonas Gonseth, chief executive, Gaerente en Hospital de Especialidades Guayaquil, Equador; Robinah Alambuya, president of the Pan African Network of People with Psychosocial Disabilities, Uganda; Daniel Iga Mwesigwa, executive medical director, Mwesigwa Medical Centre, Uganda) is available on thebmj.com.

Cite this as: BMJ 2015;350:h412

๑ BMJ Publishing Group Ltd 2015 


\section{Calls for action}

- Measure and incentivise person centred care, as defined by patients

- Trusts and clinical commissioning groups to be made accountable for maintaining integrated systems and making them easy for patients to navigate

- All patients with long term conditions to have and define their own care plans

- Open the medical record to patients and share it across providers

- Royal colleges to appoint clinical champions for person centred care

- Appoint patient officers on the trust board of every hospital

- Capitalise on new technologies to engage with patient communities and promote self care

- Include person centred care standards in education and clinical leadership

- Care Quality Commission inspections, or equivalent, to prioritise the provision of person centred care

\section{At the roundtable}

Fiona Godlee (chair), editor in chief, The BMJ

Tessa Richards, senior editor, patient partnership, The BMJ

Rosamund Snow, patient editor, The BMJ

Navjoyt Ladher, clinical editor, The BMJ

Angela Coulter, director of global initiatives, Informed Medical Decisions Foundation (www.informedmedicaldecisions.org)

Paul Wicks, vice president of innovation, PatientsLikeMe (www.patientslikeme.com)

Michael Seres, founder, 11 Health (www.11health.com)

Alf Collins, clinical associate in person centred care, Health Foundation (www.health.org.uk)

Jeremy Taylor, chief executive, National Voices (www.nationalvoices.org.uk)

Dave deBronkart, cochair, Society for Participatory Medicine (www.participatorymedicine.org)

Amir Hannan, general practitioner and member of clinical commissioning group board

Alexander Silverstein, past president, International Diabetes Federation's young leaders in diabetes project

Paul Hodgkin, founder, Patient Opinion (www.patientopinion.org.uk)

Ben Mearns, consultant in acute care and elderly medicine, Surrey and Sussex Healthcare NHS Trust

Sara Riggare, PhD student in health informatics, Karolinska Institute

Rupert Whitaker, founder, Tuke Institute (www.tukeinstitute.org)

Stephen Leyshon (observer), DNV Healthcare 